\title{
Loss of C-5 Sterol Desaturase Activity Results in Increased Resistance to Azole and Echinocandin Antifungals in a Clinical Isolate of Candida parapsilosis
}

\author{
Jeffrey M. Rybak, a C. Michael Dickens, ${ }^{\mathrm{b}}$ Josie E. Parker, ${ }^{\mathrm{c}}$ Kelly E. Caudle, ${ }^{\mathrm{a}}$ \\ Kayihura Manigaba, ${ }^{a}$ Sarah G. Whaley, ${ }^{a}$ Andrew T. Nishimoto, ${ }^{a}$

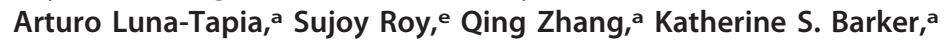 \\ Glen E. Palmer, a Thomas R. Sutter, ${ }^{\mathrm{b}}$ Ramin Homayouni,d Nathan P. Wiederhold, ${ }^{\mathrm{d}}$ \\ Steven L. Kelly, P. David Rogersa \\ Department of Clinical Pharmacy, College of Pharmacy, University of Tennessee Health Science Center, \\ Memphis, Tennessee, USAa; W. Harry Feinstone Center for Genomic Research, University of Memphis, \\ Memphis, Tennessee, USA b; Institute of Life Science, Swansea University Medical School, Swansea, Wales, \\ United Kingdomc; Bioinformatics Program, University of Memphis, Memphis, Tennessee, USAd; Fungus Testing \\ Laboratory, University of Texas Health Science Center at San Antonio, San Antonio, Texas, USAe
}

\begin{abstract}
Among emerging non-albicans Candida species, Candida parapsilosis is of particular concern as a cause of nosocomial bloodstream infections in neonatal and intensive care unit patients. While fluconazole and echinocandins are considered effective treatments for such infections, recent reports of fluconazole and echinocandin resistance in $C$. parapsilosis indicate a growing problem. The present study describes a novel mechanism of antifungal resistance in this organism affecting susceptibility to azole and echinocandin antifungals in a clinical isolate obtained from a patient with prosthetic valve endocarditis. Transcriptome analysis indicated differential expression of several genes in the resistant isolate, including upregulation of ergosterol biosynthesis pathway genes ERG2, ERG5, ERG6, ERG11,ERG24, ERG25, and $U P C 2$. Whole-genome sequencing revealed that the resistant isolate possessed an ERG3 mutation resulting in a G111R amino acid substitution. Sterol profiles indicated a reduction in sterol desaturase activity as a result of this mutation. Replacement of both mutant alleles in the resistant isolate with the susceptible isolate's allele restored wild-type susceptibility to all azoles and echinocandins tested. Disruption of ERG3 in the susceptible and resistant isolates resulted in a loss of sterol desaturase activity, high-level azole resistance, and an echinocandin-intermediate to -resistant phenotype. While disruption of ERG3 in C. albicans resulted in azole resistance, echinocandin MICs, while elevated, remained within the susceptible range. This work demonstrates that the G111R substitution in Erg3 is wholly responsible for the altered azole and echinocandin susceptibilities observed in this C. parapsilosis isolate and is the first report of an ERG3 mutation influencing susceptibility to the echinocandins.
\end{abstract}

KEYWORDS Candida, antifungal resistance, antimicrobial activity, antimicrobial agents, ergosterol, molecular genetics, mycology

andida species are among the most common causes of bloodstream infections in the United States and are associated with high rates of morbidity and mortality. While Candida albicans is the most commonly isolated causative agent of candidemia, the incidence of infections due to other non-albicans species of Candida has increased in recent decades (1-3). Of these non-albicans species, Candida parapsilosis is of particular concern as a human fungal pathogen. It exhibits the capacity to grow on a
Received 31 March 2017 Returned for modification 19 April 2017 Accepted 7 June 2017

Accepted manuscript posted online 19 June 2017

Citation Rybak JM, Dickens CM, Parker JE, Caudle KE, Manigaba K, Whaley SG, Nishimoto AT, Luna-Tapia A, Roy S, Zhang Q, Barker KS, Palmer GE, Sutter TR, Homayouni R, Wiederhold NP, Kelly SL, Rogers PD. 2017. Loss of $\mathrm{C}-5$ sterol desaturase activity results in increased resistance to azole and echinocandin antifungals in a clinical isolate of Candida parapsilosis. Antimicrob Agents Chemother 61:e00651-17. https://doi.org/10.1128/AAC $.00651-17$

Copyright $\odot 2017$ American Society for Microbiology. All Rights Reserved. Address correspondence to P. David Rogers, progers3@uthsc.edu.

* Present address: Kelly E. Caudle, Department of Pharmaceutical Sciences, St. Jude Research Hospital, Memphis, Tennessee, USA. 
wide variety of surfaces, such as prosthetic materials and intravascular devices, and grows well within parenteral nutrition. It persists in hospital environments and may be transmitted nosocomially via hand carriage. Low-weight neonates and intensive care patients are among those at the highest risk of infections with C. parapsilosis (4). Relative to what is known about the mechanisms by which antifungal drug resistance develops in C. albicans, there is little information regarding the mechanisms by which antifungal drug resistance develops in C. parapsilosis. Uniquely among Candida species, C. parapsilosis demonstrates intrinsic reduced in vitro susceptibility to the echinocandins, presumably as a result of a naturally occurring polymorphism in FKS1 (5). While it is uncommon, clinical resistance to this class of antifungals has emerged in C. parapsilosis $(6,7)$. Azole resistance, on the other hand, is more common in C. parapsilosis, in which the rates of fluconazole resistance are approximately five times higher than those in C. albicans (8). Recent reports indicate that overexpression of the drug efflux pump Mdr1 as well as an increase in or mutation of the target of the azoles, Erg11, contributes to azole resistance in this species (9-12).

In this study, we investigated the mechanisms underlying antifungal drug resistance in a clinical isolate of $C$. parapsilosis by comparing it to a genetically matched, antifungal-susceptible isolate from the same patient. Through whole-transcriptome and whole-genome sequencing (WGS) analysis and allelic replacement, we demonstrate that a mutation in ERG3 leads to resistance to the azole antifungals via alternate sterol production. Additionally, we discover that this mutation in ERG3, as well as its disruption, also causes increased resistance to the echinocandins. This is the first report of an ERG3-related resistance mechanism in C. parapsilosis and is the first evidence in any Candida species that such mutations also influence susceptibility to the echinocandins.

\section{RESULTS}

Clinical isolates and susceptibility testing. The C. parapsilosis isolates used in this study were collected over the course of multiple hospitalizations from a patient with aortic valve endocarditis, as outlined elsewhere (13). These isolates were kindly provided by Jose Vazquez. The originally reported antifungal susceptibilities (determined using the methodologies previously recommended in National Committee for Clinical Laboratory Standards document M27-A2 [14]) both for the susceptible isolate collected at the initial admission (isolate 1 ) and for the subsequently collected resistant isolate (isolate 2) for several azoles, echinocandins, and amphotericin B are shown in Table 1. MIC values for fluconazole, itraconazole, voriconazole, posaconazole, caspofungin, anidulafungin, micafungin, and amphotericin B were independently determined for this study by the Fungus Testing Laboratory at the University of Texas Health Science Center at San Antonio (Table 1). Isolate 1 was susceptible to all azoles tested when the result was read at both 24 and $48 \mathrm{~h}$, and likewise, it was susceptible to amphotericin $B$ and all echinocandins tested (when susceptibility was determined at 24 h). Interestingly, when susceptibility was determined at $24 \mathrm{~h}$, isolate 2 appeared to be susceptible to all azoles tested, whereas when susceptibility was determined at $48 \mathrm{~h}$, isolate 2 was resistant to all azoles tested. These results were consistent upon repeat testing. Isolate 2 also exhibited an increase in MIC for the echinocandins, reaching the intermediate range for micafungin and anidulafungin. No change in susceptibility to amphotericin $B$ was observed. In separate experiments, growth curves for fluconazole MIC cultures were plotted at 24 and $48 \mathrm{~h}$ (Fig. 1). At both time points, isolate 2 phenocopied the $\operatorname{erg} 3 \Delta / \operatorname{erg} 3 \Delta$ mutants.

Transcriptional profiling reveals increased expression of ergosterol biosynthesis genes. Global changes in gene expression between isolates 1 and 2 were determined by RNA sequencing. A total of 378 genes (see Table S1 in the supplemental material) were observed to be reproducibly upregulated by a minimum of 1.5 -fold in the resistant isolate compared to their level of expression in the susceptible isolate. Of note, only three of these genes (UPC2, ATC1, and CPAR2_400860) have been characterized in C. parapsilosis at this time. Gene Ontology term analysis performed using the 
TABLE 1 Antifungal susceptibilities of C. albicans and C. parapsilosis isolates to azoles, echinocandins, and amphotericin B

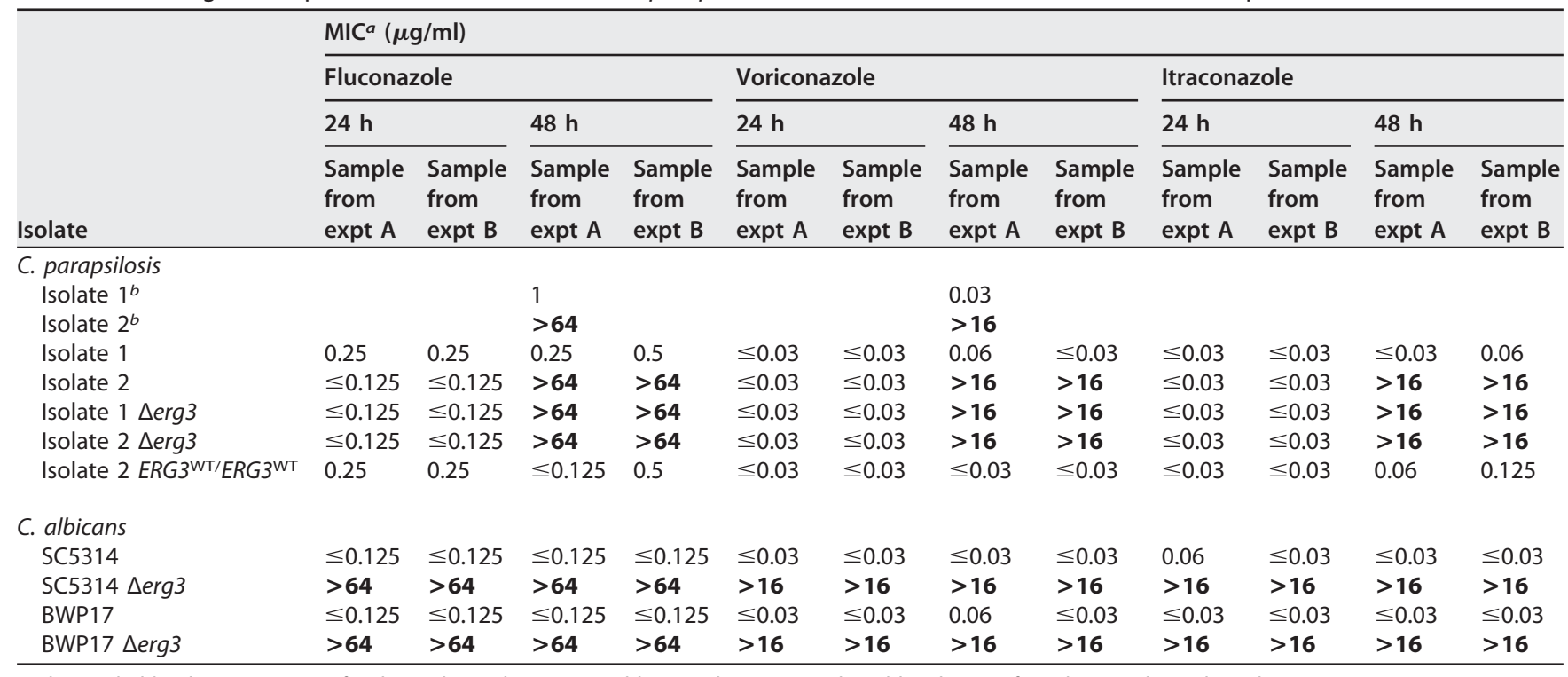

aValues in bold indicate resistance for the azoles and nonsusceptible according to CLSI clinical breakpoints for echinocandin and amphotericin B.

${ }^{b} \mathrm{MIC}$ values were reported previously (13) and were determined using the methodologies recommended in National Committee for Clinical Laboratory Standards document M27-A2 (14).

Candida Genome Database (www.candidagenome.org) revealed genes of the sterol metabolic process to be the most significantly enriched among all upregulated genes $\left(P=2.96 \mathrm{e}^{-5}\right)$. Of these 14 genes, as shown in Table 2 , the sterol regulatory transcription factor UPC2 and the C. parapsilosis orthologs of identified C. albicans UpC2 (CaUpc2) target genes (ERG1, ERG2, ERG5, ERG6, ERG11, ERG24, and ERG25) were included (15). Differential expression of key ergosterol biosynthesis genes, as well as the lack of a change in expression of the multidrug transporter genes CDR1, CDR2, and $M D R 1$, was confirmed by quantitative reverse transcription-PCR (data not shown). An additional 32 genes were observed to be upregulated in the resistant isolate, but the extent of upregulation could not be reliably quantified due to the extremely low transcript counts for the susceptible isolate (Table S2). Genes which were reproducibly downregulated in the resistant isolate compared to their level of expression in the susceptible isolate are listed in Table S3.

Next-generation sequencing identified an SNP in ERG3 in the resistant isolate. To further characterize this matched isolate pair, whole-genome sequencing was used to identify single nucleotide polymorphisms (SNPs) occurring in isolate 2 compared to the sequence of isolate 1 (Fig. 2). A total of 462 SNPs involving 305 individual genes was detected. Many of the genes containing SNPs are involved in cell adhesion, biofilm formation, and cytoskeletal rearrangement. A homozygous nonsynonymous mutation was detected in ERG3, in which a glycine was replaced by an arginine at position 111. This polymorphism was confirmed by Sanger-based sequencing (data not shown).

Analysis of copy number variation and loss of heterozygosity. The Yeast Mapping Analysis Pipeline (Ymap; lovelace.cs.umn.edu/Ymap) was used to identify chromosomal copy number variation (CNV) and loss of heterozygosity (LOH) between isolate 1 and isolate 2 (16). CNV and SNP analyses of isolate 1 revealed no segmental or chromosomal CNV and scattered regions of increased heterozygosity across each chromosome (Fig. 3A). Using isolate 1 as a parental reference isolate, the WGS data for isolate 2 were then assessed. Unlike its parent isolate, isolate 2 exhibited segmental tetraploidy in a single chromosome (Contig005809). In this region, containing 113 genes (Table S4), complete duplication across approximately 233,000 bases was observed (Fig. 3B). Additionally, LOH analysis revealed that while relatively little allelic variation was present in isolate 1, much of this variation was lost in isolate 2 (Fig. S1). 
TABLE 1 (Continued)

\begin{tabular}{|c|c|c|c|c|c|c|c|c|c|c|c|c|c|c|}
\hline \multicolumn{15}{|c|}{$\mathrm{MIC}^{a}(\mu \mathrm{g} / \mathrm{ml})$} \\
\hline \multicolumn{4}{|c|}{ Posaconazole } & \multirow{2}{*}{\multicolumn{2}{|c|}{$\frac{\text { Anidulafungin }}{24 \mathrm{~h}}$}} & & \multirow{2}{*}{\multicolumn{2}{|c|}{$\frac{\text { Caspofungin }}{24 \mathrm{~h}}$}} & & \multicolumn{3}{|c|}{ Micafungin } & \multirow{2}{*}{\multicolumn{2}{|c|}{$\begin{array}{l}\text { Amphotericin B, } \\
24 \mathrm{~h}\end{array}$}} \\
\hline \multicolumn{2}{|l|}{$24 \mathrm{~h}$} & \multicolumn{2}{|l|}{$48 \mathrm{~h}$} & & & & & & & $24 \mathrm{~h}$ & & & & \\
\hline $\begin{array}{l}\text { Sample } \\
\text { from } \\
\text { expt A }\end{array}$ & $\begin{array}{l}\text { Sample } \\
\text { from } \\
\text { expt B }\end{array}$ & $\begin{array}{l}\text { Sample } \\
\text { from } \\
\text { expt A }\end{array}$ & $\begin{array}{l}\text { Sample } \\
\text { from } \\
\text { expt B }\end{array}$ & $\begin{array}{l}\text { Sample } \\
\text { from } \\
\text { expt A }\end{array}$ & $\begin{array}{l}\text { Sample } \\
\text { from } \\
\text { expt B }\end{array}$ & $48 \mathrm{~h}$ & $\begin{array}{l}\text { Sample } \\
\text { from } \\
\text { expt A }\end{array}$ & $\begin{array}{l}\text { Sample } \\
\text { from } \\
\text { expt B }\end{array}$ & $48 \mathrm{~h}$ & $\begin{array}{l}\text { Sample } \\
\text { from } \\
\text { expt A }\end{array}$ & $\begin{array}{l}\text { Sample } \\
\text { from } \\
\text { expt B }\end{array}$ & $48 \mathrm{~h}$ & $\begin{array}{l}\text { Sample } \\
\text { from } \\
\text { expt A }\end{array}$ & $\begin{array}{l}\text { Sample } \\
\text { from } \\
\text { expt B }\end{array}$ \\
\hline & & & & & & 1 & & & 2 & & & 8 & 0.25 & \\
\hline & & & & & & 2 & & & $>16$ & & & $>16$ & 0.5 & \\
\hline$\leq 0.03$ & $\leq 0.03$ & $\leq 0.03$ & $\leq 0.03$ & 2 & 2 & & 1 & 1 & & 2 & 2 & & 0.5 & 0.5 \\
\hline$\leq 0.03$ & $\leq 0.03$ & $>16$ & $>16$ & 4 & 4 & & 2 & 1 & & 4 & 4 & & 0.5 & 0.5 \\
\hline$\leq 0.03$ & $\leq 0.03$ & $>16$ & $>16$ & 4 & 4 & & 2 & 2 & & 8 & 8 & & 0.5 & 1 \\
\hline$\leq 0.03$ & $\leq 0.03$ & $>16$ & $>16$ & 4 & 4 & & 2 & 2 & & 4 & 4 & & 0.5 & 0.5 \\
\hline$\leq 0.03$ & $\leq 0.03$ & 0.06 & $\leq 0.03$ & 2 & 2 & & 1 & 0.5 & & 2 & 2 & & 0.25 & 0.5 \\
\hline$\leq 0.03$ & $\leq 0.03$ & $\leq 0.03$ & $\leq 0.03$ & $\leq 0.015$ & $\leq 0.015$ & & 0.25 & 0.25 & & 0.03 & 0.03 & & 0.25 & 0.5 \\
\hline$>16$ & $>16$ & $>16$ & $>16$ & 0.06 & 0.06 & & 0.25 & 0.5 & & 0.06 & 0.06 & & 0.5 & 0.5 \\
\hline 0.125 & $\leq 0.03$ & 0.06 & $\leq 0.03$ & $\leq 0.015$ & $\leq 0.015$ & & 0.25 & 0.25 & & 0.03 & 0.03 & & 0.25 & 0.5 \\
\hline$>16$ & $>16$ & $>16$ & $>16$ & 0.06 & 0.06 & & 0.25 & 0.25 & & 0.06 & 0.06 & & 0.5 & 0.5 \\
\hline
\end{tabular}

Loss of Erg3 activity confers increased resistance to echinocandins in C. parapsilosis and, to a lesser extent, C. albicans. We first deleted both alleles of ERG3 in both the susceptible (isolate 1) and the resistant (isolate 2) isolates. As expected, the loss of ERG3 resulted in resistance to all azoles tested (Table 1). Surprisingly, deletion of ERG3 also resulted in MICs that fell within the intermediate to resistant range for the echinocandins (Table 1). In order to determine if this also occurs in the related species C. albicans, we tested the echinocandin susceptibilities of two independent ERG3 deletion mutants constructed in two different backgrounds. In both cases, deletion of ERG3 resulted in negligible increases in echinocandin MICs (Table 1).

The G111R substitution in Erg3 confers reduced susceptibility to azoles and echinocandins. In order to directly assess the role of the G111R amino acid substitution in Erg3 in the reduced susceptibility to azoles and echinocandins, we introduced the wild-type (WT) ERG3 alleles from the susceptible isolate (isolate 1) into its resistant counterpart (isolate 2), creating a homozygous mutant, and performed testing for susceptibility to azoles and echinocandins. Introduction of the wild-type ERG3 alleles into isolate 2 restored susceptibility to all azoles tested (Table 1). Surprisingly, susceptibility to the echinocandins was also restored to the levels observed in isolate 1.

The G111R substitution in Erg3 leads to alterations in sterol biosynthesis. As the mutation in ERG3 is not a nonsense mutation, we questioned if, alternatively, the activity of Erg3 is reduced by this mutation, leading instead to a less functional protein. Perturbations in this protein would be evident with increases in accumulated ergosta7,22-dienol, episterol, and ergosta-7-enol. Sterol profiles were obtained using gas chromatography (GC)-mass spectrometry (MS) and are shown in Table 3. Isolate 1 exhibited a reasonably normal sterol profile in which ergosterol comprised the highest percentage. On the other hand, isolate 2 exhibited profiles in which ergosta-7-enol and ergosta-7,22-dienol represented the largest portion of the total cell sterol fraction. This is consistent with a reduction in sterol desaturase activity. Upon replacement of the mutant ERG3 alleles in isolate 2 with the wild-type allele from isolate 1, normal sterol profiles were restored. Deletion of ERG3 in both isolate 1 (susceptible isolate) and isolate 2 (resistant isolate) resulted in profiles consistent with the complete loss of Erg3 activity.

\section{DISCUSSION}

Most of what is known about the genetic basis of antifungal resistance has been determined from $C$. albicans. Mechanisms of azole resistance in this species include 
A.

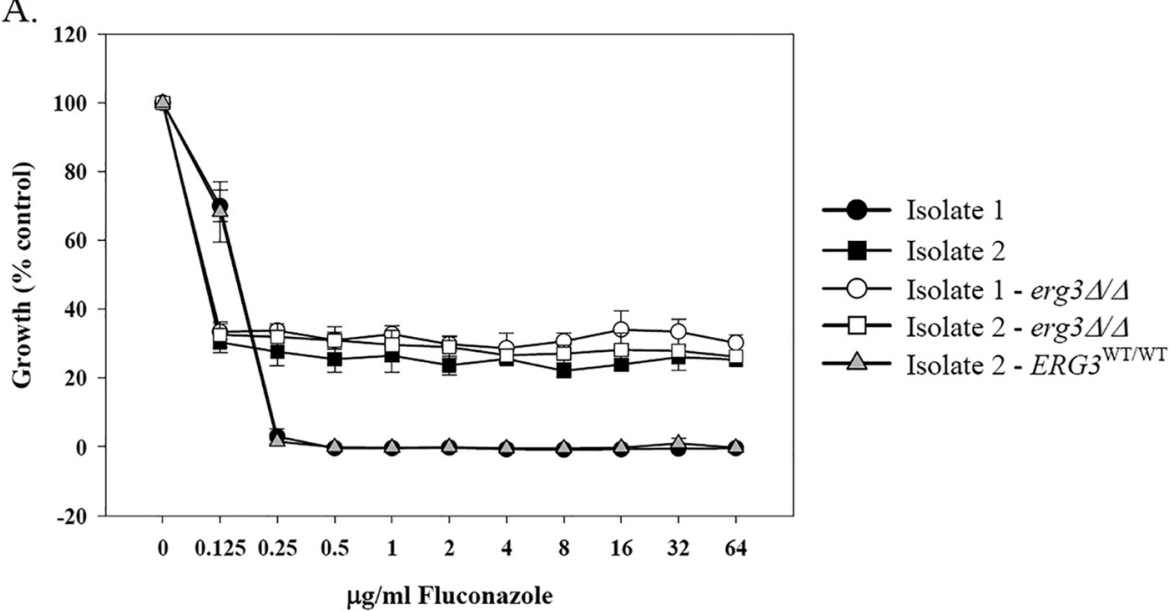

B.

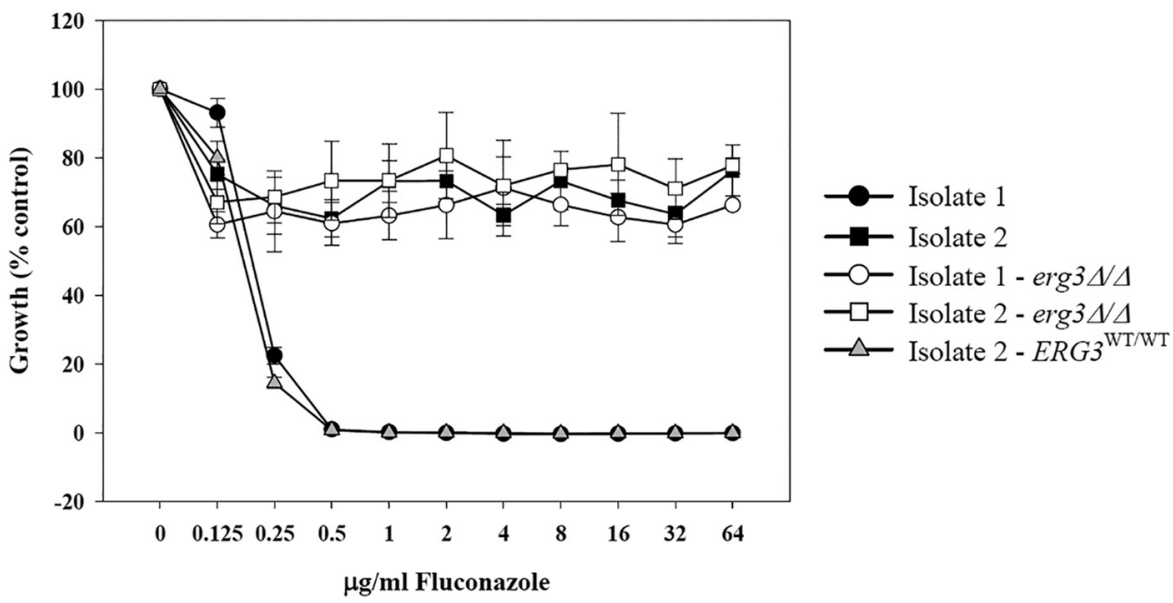

FIG 1 Fluconazole MIC growth curves. Isolates 1 and 2 and their related strains were grown for $24 \mathrm{~h}(\mathrm{~A})$ and $48 \mathrm{~h}(\mathrm{~B})$ in the presence of the indicated concentrations of fluconazole.

overexpression of the gene encoding the target enzyme, Erg11, and nonsynonymous mutations in both ERG11 and ERG3. Additionally, upregulation of drug efflux pumps represents an additional mechanism of azole resistance; $A B C$ transporters C $\mathrm{dr} 1$ and $\mathrm{Cdr} 2$ confer resistance to multiple azoles, while the major facilitator superfamily transporter Mdr1 confers resistance to fluconazole, ketoconazole, and voriconazole (17). Echinocandins, on the other hand, function by inhibiting $\beta$-D-glucan synthase activity, encoded by FKS1 in C. albicans and FKS1 and FKS2 in Saccharomyces cerevisiae (18). Although mutations within FKS genes have been linked to echinocandin resistance in several species of Candida (19), there are also additional mechanisms by which this resistance may occur. These include the activation of signaling pathways which regulate the stress response and cell wall integrity, as well as synthesis of ancillary cell wall components, such as chitin and mannan (20).

In the present study, RNA sequencing revealed the genes that are differentially expressed between these two genetically related clinical isolates. We did not observe overexpression of any gene encoding a characterized multidrug transporter, suggesting that azole resistance was unlikely due to reduced intracellular accumulation of drug. Furthermore, the observed 1.6- to 2.6-fold increased expression of ERG11 in the resistant isolate compared to its level of expression in the susceptible isolate alone would not be expected to produce high-level pan-azole resistance, particularly in the absence of an accompanying ERG11 mutation. Lastly, we did not observe any change in gene expression that would indicate a root cause for the observed reduction in 
TABLE 2 Genes involved in sterol metabolic processes overrepresented among the upregulated genes in isolate 2 relative to isolate 1

\begin{tabular}{|c|c|c|c|c|c|}
\hline \multirow[b]{2}{*}{ Gene } & \multicolumn{3}{|l|}{ Gene name ${ }^{a}$} & \multicolumn{2}{|l|}{ Fold change $^{b}$} \\
\hline & C. parapsilosis & S. cerevisiae & C. albicans & $\begin{array}{l}\text { Sample from } \\
\text { expt } A\end{array}$ & $\begin{array}{l}\text { Sample from } \\
\text { expt B }\end{array}$ \\
\hline CPAR2_601530 & & GRE2 & & 4.8 & 5.0 \\
\hline CPAR2_201490 & & & & 7.2 & 1.7 \\
\hline CPAR2_405010 & & ERG6 & ERG6 & 2.3 & 3.8 \\
\hline CPAR2_202280 & & $D A P 1$ & $D A P 1$ & 3.3 & 1.6 \\
\hline CPAR2_405900 & & ERG24 & ERG24 & 2.0 & 2.7 \\
\hline CPAR2_103490 & & ATF2 & & 2.3 & 2.2 \\
\hline CPAR2_801560 & & $E R G 27$ & $E R G 27$ & 2.2 & 2.2 \\
\hline CPAR2_303740 & ERG11 & ERG11 & ERG11 & 1.6 & 2.6 \\
\hline CPAR2_801410 & & ERG25 & ERG25 & 1.8 & 2.2 \\
\hline CPAR2_105000 & & YEH1 & & 2.0 & 2.0 \\
\hline CPAR2_210480 & & ERG1 & ERG1 & 1.7 & 2.2 \\
\hline CPAR2_207280 & UPC2 & UPC2 & UPC2 & 1.8 & 1.8 \\
\hline CPAR2_703970 & & ERG5 & ERG5 & 1.6 & 1.6 \\
\hline CPAR2_109890 & & ERG2 & ERG2 & 1.6 & 1.5 \\
\hline
\end{tabular}

${ }^{a}$ Genes shown in bold have orthologs regulated by CaUPC2.

${ }^{b}$ Fold change values represent those for genes upregulated in isolate 2 a minimum of 1.5 -fold compared to their level of expression in isolate 1 in samples from independent experiments $A$ and $B$.

echinocandin susceptibility, such as an increase in glucan synthase or chitin synthase expression.

Notably among the upregulated genes are several involved in the ergosterol biosynthesis pathway. This biosynthetic pathway results in the production of an essential fungal membrane component, ergosterol, and inhibition of the azole target sterol demethylase leads to the production of toxic sterol intermediates (21). The azoles exploit this and function specifically by inhibiting the enzyme $14 \alpha$-lanosterol demethylase, encoded by ERG11, leaving the cell unable to produce ergosterol (22). The genes which were observed to be upregulated within the resistant isolate, ERG1, ERG2, ERG5, $E R G 6, E R G 11, E R G 24, E R G 25, E R G 27$, and DAP1, encode various proteins involved in sterol processing within this pathway and are located both upstream and downstream of the azole target, Erg11. Additionally, the gene encoding the sterol regulatory transcription factor Upc2 was upregulated. These observations are consistent with a loss of sterol desaturase activity, leading to decreased membrane ergosterol and activation of Upc2. In this way, upregulation of these critical genes represents a response to facilitate increased conversion of lanosterol to ergosterol in the event that production declines or is disrupted. This is further supported by the results of whole-genome sequencing of the isolate pair. Among the relatively small number of the SNPs detected in the resistant isolate which did not occur in the susceptible isolate, a nonsynonymous mutation was identified in ERG3. This gene is located downstream in the ergosterol biosynthesis pathway relative to the positions of the genes found to be upregulated by RNA sequencing. The amino acid substitution, at position 111 of the predicted protein, changes a glycine to an arginine.

A mutation within ERG3 has been documented in clinical isolates as well as passagederived isolates of C. albicans and linked to both amphotericin B resistance and high-level azole resistance $(23,24)$. When azoles inhibit the function of $14 \alpha$ demethylase and, therefore, the production of ergosterol, ERG3 encodes an enzyme which converts the nontoxic $14 \alpha$-methylated sterol intermediates into the toxic sterol $14 \alpha$-methyl-ergosta-8,24(28)-dien-3,6-diol. A reduction in or loss of this enzyme results instead in the accumulation of nontoxic ergosta-7,22-dienol. This leads to high levels of resistance to the azole drug class.

The loss of ergosterol in the yeast cell membrane, the target of amphotericin B, has been reported to result in resistance to this antifungal (25-27). However, using standard CLSI methods, we did not observe a reproducible increase in the amphotericin B MIC at 24 or $48 \mathrm{~h}$ for the C. parapsilosis or C. albicans mutants deleted for ERG3 or for the 


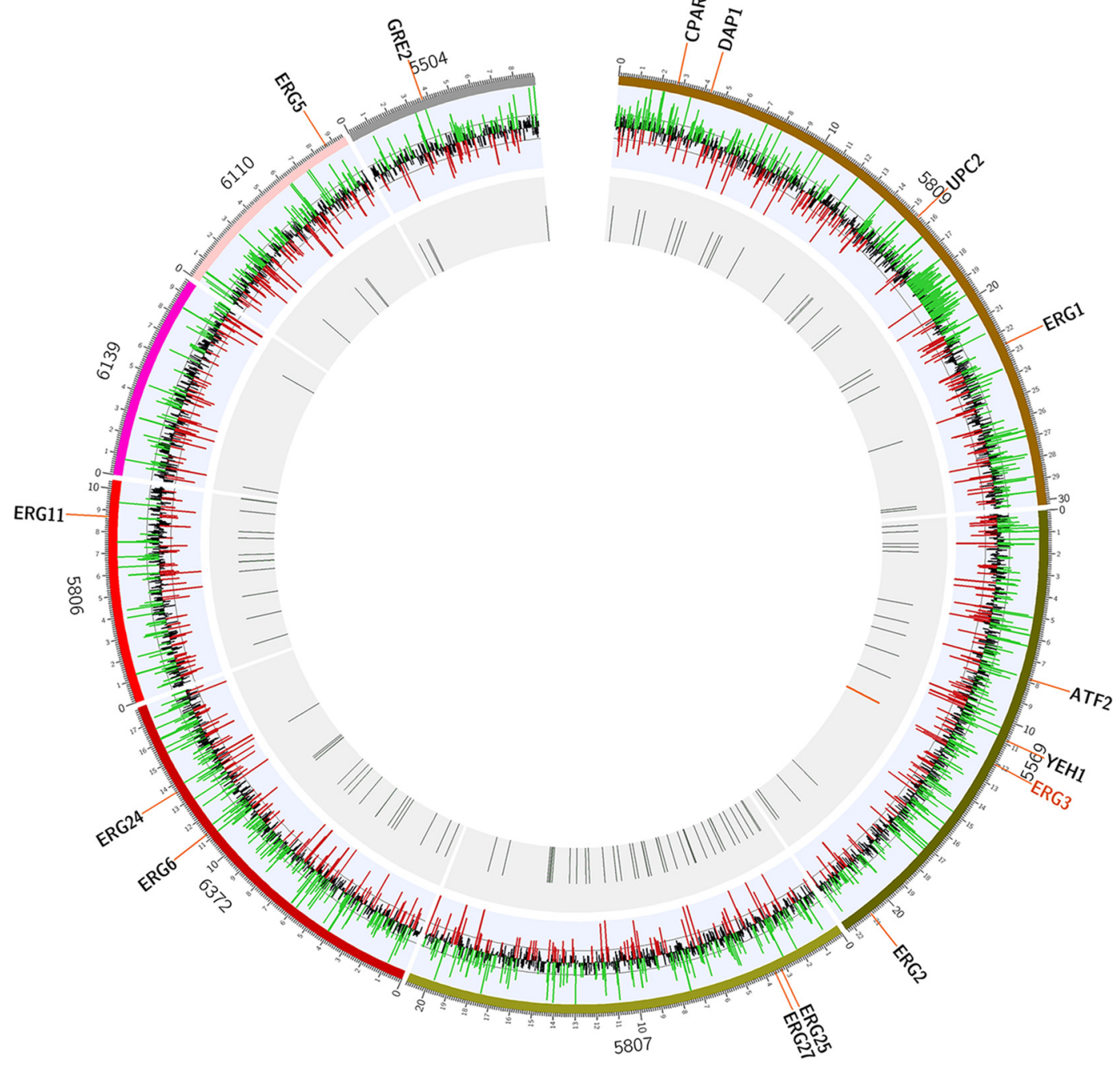

FIG 2 Schematic representation of RNA and whole-genome sequencing data for isolate 2 compared to isolate 1. Colored circle segments represent contig boundaries. The outer track represents RNA sequence data, such that the green lines indicate those genes which were overexpressed in isolate 2 by at least 1.5 -fold relative to their levels of expression in isolate 1 and red lines indicate those genes which were underexpressed by 0.5 -fold or less relative to their levels of expression in isolate 1 . The inner track represents sequence variants which were found in isolate 2 but not in isolate 1 for the whole-genome sequence data. Overexpressed genes of the ergosterol biosynthetic process are labeled with $S$. cerevisiae ortholog gene names.

C. parapsilosis mutant expressing the G111R mutation. The majority of ERG3 mutations reported in the literature result in premature stop codons; however, there have been cases in which the strains instead exhibit nonsynonymous mutations, as observed in the isolate pair evaluated in the present study $(23,28-30)$.

The finding that the G111R amino acid substitution also explained the reduced susceptibility to the echinocandins observed in this isolate was unexpected, as, until now, alterations in sterol desaturase activity have not been associated with this phenotype. It is important to note that deletion of ERG3 had a similar effect on echinocandin susceptibility in C. parapsilosis, but this was not the case when ERG3 was deleted in C. albicans. It is possible that a mutation in ERG3 that impairs sterol desaturase activity, combined with the naturally occurring polymorphism in FKS1 in C. parapsilosis, uniquely impacts echinocandin susceptibility in this Candida species.

Our finding of an ERG3 mutation as a cause of azole resistance in this clinical isolate is supported by the recent work of Branco et al. (31). In that study, a C. parapsilosis strain 


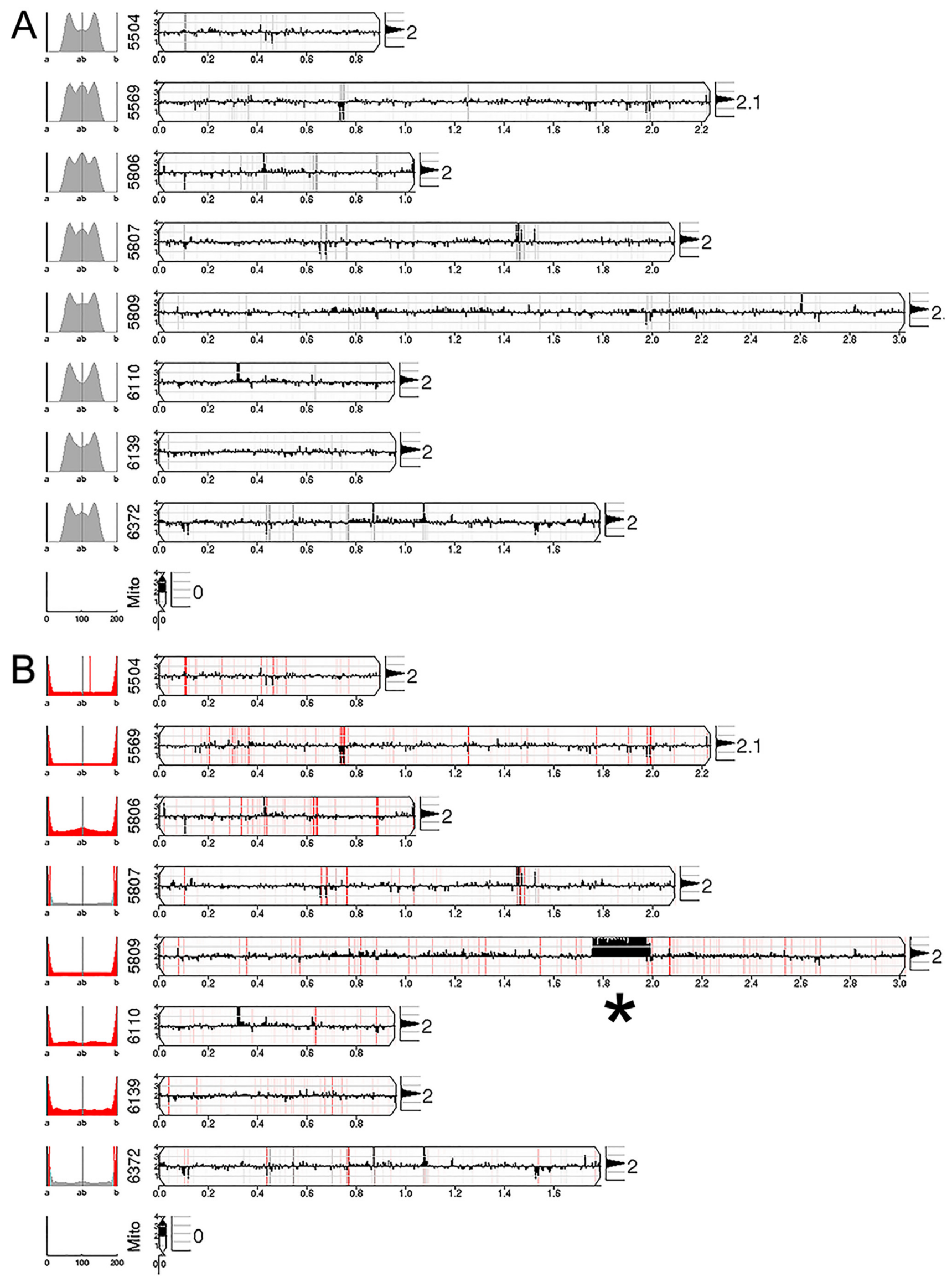


TABLE 3 Sterol composition of each isolate and each ERG3 mutant

\begin{tabular}{|c|c|c|c|c|}
\hline \multirow[b]{2}{*}{ Sterol } & \multicolumn{4}{|c|}{ Amt of sterol as \% of total sterols } \\
\hline & Isolate 1 & Isolate 2 & $\begin{array}{l}\text { Isolate } 1 \\
\operatorname{erg} 3 \Delta / \operatorname{erg} 3 \Delta\end{array}$ & $\begin{array}{l}\text { Isolate } 2 \\
\text { ERG3 }\end{array}$ \\
\hline Ergosta-5,7,24(28)-tetranol & 4.0 & 0.5 & 0.4 & 0.5 \\
\hline Unknown sterol with $\mathrm{m} / \mathrm{z} 470$ & & & 7.0 & \\
\hline Zymosterol & 4.2 & 7.5 & & 1.9 \\
\hline Ergosterol & 65.4 & 9.1 & & 51.8 \\
\hline Ergosta-7,22-dienol & 9.3 & 71.7 & 76.8 & 22.3 \\
\hline Fecosterol [E8, 24(28)] & 1.4 & 0.6 & 0.9 & 0.7 \\
\hline Ergosta-8-enol & & 0.8 & 1.2 & 0.6 \\
\hline Ergosta-5,7-dienol & 6.3 & 0.4 & 0.3 & 8.6 \\
\hline Episterol [E7, 24(28)] & 3.2 & 3.3 & 4.2 & 3.2 \\
\hline Ergosta-7-enol & 1.9 & 4.2 & 8.4 & 6.6 \\
\hline Lanosterol/obtusifoliol & 2.8 & 0.5 & 0.3 & 2.7 \\
\hline 4,4,-Diemthyl cholesta-8,24-dienol & 1.7 & 1.4 & 0.6 & 1.1 \\
\hline
\end{tabular}

that was evolved to become resistant to posaconazole in the laboratory was found to have a mutation in ERG3 (R135I) similar to the mutation found in the present study that resulted in resistance to fluconazole, voriconazole, and posaconazole. Echinocandin susceptibilities, however, were not reported.

The matched isolate pair described here provided an opportunity to evaluate factors contributing to a unique case of antifungal resistance. We reasoned that the mechanism(s) underlying this resistance would be detectable by differences in gene expression and/or by whole-genome sequence analysis. Here we have demonstrated that a mutation in ERG3, which encodes a key enzyme for the production of the membrane sterol ergosterol, increases resistance not only to the azoles but also to the echinocandins in a clinical isolate. This is the first report of a mutation in ERG3 influencing susceptibility to the echinocandins and of a single mechanism that affects susceptibility to these two important classes of antifungals.

\section{MATERIALS AND METHODS}

Strains and media. All C. parapsilosis isolates used in this study are listed in Table 4. Isolates were kept as frozen stocks in $40 \%$ glycerol at $-80^{\circ} \mathrm{C}$ and subcultured on YPD ( $1 \%$ yeast extract, $2 \%$ peptone, $1 \%$ dextrose) agar plates at $30^{\circ} \mathrm{C}$. YPD liquid medium was used for routine growth of the strains, while YPM (1\% yeast extract, 2\% peptone, 1\% maltose) liquid medium was used for induction of the MAL2 promoter in constructed strains. Nourseothricin $(200 \mu \mathrm{g} / \mathrm{ml})$ was added to YPD agar plates for selection of isolates containing the SAT1-flipper cassette (32). One Shot Escherichia coli TOP10 chemically competent cells (Invitrogen) were used for plasmid construction. These strains were grown in Luria-Bertani (LB) broth or on LB agar plates supplemented with $100 \mu \mathrm{g} / \mathrm{ml}$ ampicillin (Sigma) or $50 \mu \mathrm{g} / \mathrm{ml} \mathrm{kanamycin}$ (Fisher BioReagents), when needed.

Drug susceptibility testing. Drug susceptibility testing was performed by broth microdilution according to reference standard methods described in CLSI document M27-A3 (33). Testing was performed at least in duplicate for each isolate and each antifungal agent. The starting inoculum was between $0.5 \times 10^{3}$ and $2.5 \times 10^{3}$ cells $/ \mathrm{ml}$, and all testing was performed in RPMI 1640 with $0.2 \%$ glucose buffered with $0.165 \mathrm{M}$ MOPS (morpholinepropanesulfonic acid) and adjusted to $\mathrm{pH}$ 7.0. The plates were incubated at $35^{\circ} \mathrm{C}$, and MICs were read visually for the echinocandins and azoles and were considered the lowest concentration that resulted in 50\% inhibition of growth compared to that for the drug-free growth control at both 24 and $48 \mathrm{~h}$. For amphotericin B, the MIC was read as the lowest concentration that resulted in complete inhibition of growth at each of these time points.

The susceptibility tests with fluconazole were also repeated, and the results were verified by broth microdilution using a microplate spectrophotometer with optical density readings as the endpoint. Each strain was tested at least in triplicate. Cultures were diluted to $2.5 \times 10^{3}$ cells $/ \mathrm{ml}$ in sterile RPMI 1640 (Sigma, St. Louis, MO) with $2 \%$ glucose buffered with 0.165 M MOPS and adjusted to $\mathrm{pH}$ 7.0. Plates were incubated at $35^{\circ} \mathrm{C}$ for 24 and $48 \mathrm{~h}$ with shaking. The optical density at $600 \mathrm{~nm}$ was read with a BioTek Synergy 2 microplate reader (Fisher Scientific, Waltham, MA); the background reading due to the medium was subtracted from all readings. The relative growth was calculated as the percentage of cell

FIG 3 Horizontal tracks represent the C. parapsilosis contig as labeled. The black line running horizontally through each contig represents the predicted local copy number, with the center set being equal to 2 and divergences up or down indicating an increased or decreased copy number at that locus, respectively. A region of segmental tetraploidy is denoted with an asterisk. (A) Gray vertical lines represent local allelic variation, with the darker gray indicating higher concentrations of heterozygosity. (B) Red vertical lines represent a loss of heterozygosity relative to that for the parent isolate (isolate 1 ), with darker red indicating a greater degree of change in heterozygosity. 
TABLE 4 C. parapsilosis strains and isolates used in this study

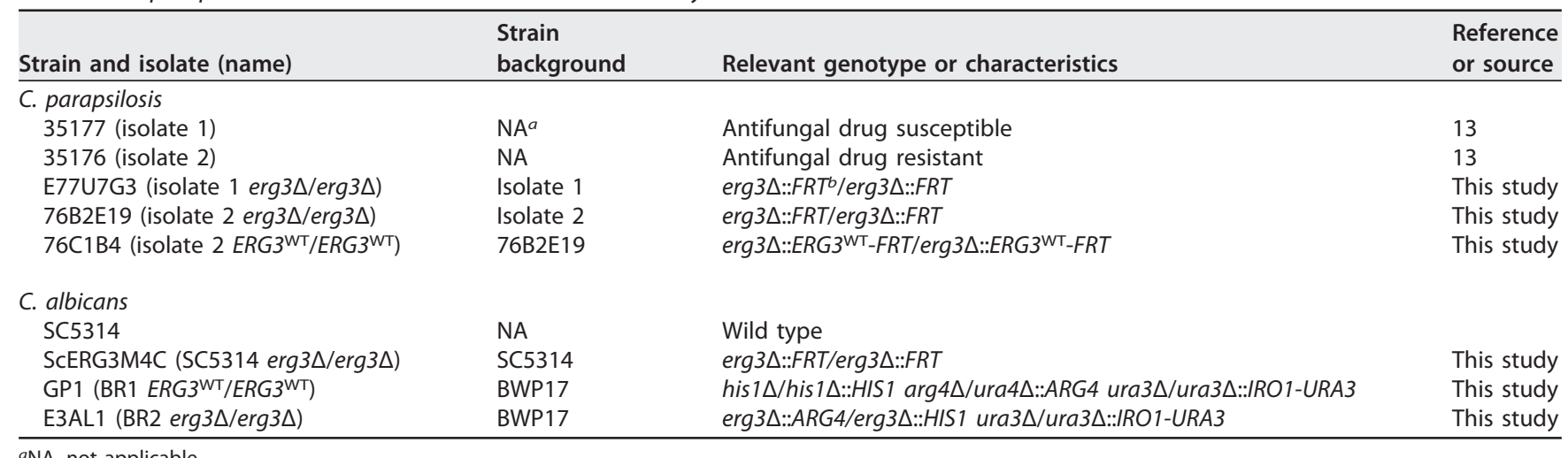

aNA, not applicable.

${ }^{b} \mathrm{FRT}$, FLP recombination target.

growth in drug-containing medium relative to the cell growth in the absence of drug; the results were plotted as percent inhibition versus the fluconazole concentration.

Construction of plasmids. All primers used are listed in Table 5. An ERG3 deletion construct for $C$. parapsilosis was generated by amplifying an Apal-Xhol-containing fragment consisting of flanking regions upstream from positions -280 to +51 relative to the start codon of C. parapsilosis ERG3 using primers ERG3-A and ERG3-B, as well as a Notl-Sacll-containing fragment consisting of downstream flanking regions from positions +1047 to +1868 using primers ERG3-C and ERG3-D. These upstream and downstream fragments of ERG3 were cloned upstream and downstream, respectively, of the SAT1-flipper cassette in plasmid PSFS2 to result in plasmid p77ERG3. Additionally, the coding region of ERG3 was amplified from either isolate 1 or isolate 2 with primers ERG3-A and ERG3-E. Each of these Apal-Xholcontaining fragments replaced the upstream sequence in cassette p77ERG3 to introduce the entire gene, creating plasmids p77ERG3comp and p76ERG3.

Similarly, an ERG3 deletion construct for C. albicans was generated by amplifying an Apal-Xholcontaining fragment consisting of flanking regions upstream from positions -350 to +39 relative to the start codon of C. albicans ERG3 using primers CaERG3A-F and CaERG3B-R, as well as a Notl-Sacllcontaining fragment consisting of flanking regions from positions +997 to +1677 downstream of the start codon using primers CaERG3C-F and CaERG3D-R. These upstream and downstream fragments were cloned upstream and downstream, respectively, of the SAT1-flipper cassette in plasmid pSFS2, resulting in pCaERG3M1.

Candida parapsilosis transformation. The C. parapsilosis strains were transformed by electroporation as described previously but with some modifications (32). Cells were grown for $6 \mathrm{~h}$ in $2 \mathrm{ml}$ YPD liquid medium, and then $4 \mu \mathrm{l}$ of this cell suspension was passed to $50 \mathrm{ml}$ of fresh YPD liquid medium and grown overnight at $30^{\circ} \mathrm{C}$ in a shaking incubator. When the culture's optical density at $600 \mathrm{~nm}$ reached 2.0, cells were collected by centrifugation, resuspended in $1 \mathrm{ml} 10 \times$ TE (Tris-EDTA) buffer, $1 \mathrm{ml}$ lithium

TABLE 5 Primers used in this study

\begin{tabular}{|c|c|}
\hline $\begin{array}{l}\text { Primer purpose and primer } \\
\text { name }\end{array}$ & Sequence $^{a}$ \\
\hline \multicolumn{2}{|c|}{$\begin{array}{l}\text { C. parapsilosis gene } \\
\text { disruption/complementation }\end{array}$} \\
\hline ERG3-A & 5'-GACAAACAAAATAAGGGCCCAAAATTAAAGG-3' \\
\hline ERG3-B & 5'-AGCATATAGTCTCTCGAGTAGGTAATAAT-3' \\
\hline ERG3-C & 5'-GATAGCCGCGGAAGATCATACAGAAGAC-3' \\
\hline ERG3-D & 5'-AAAATAGAGCTCCTGGGTGGGAATTAT - $3^{\prime}$ \\
\hline ERG3-E & 5'-AAAATACAGTTACTCGAGGGGAATTAT-3' \\
\hline \multicolumn{2}{|l|}{ C. parapsilosis ERG3 sequencing } \\
\hline ERG3-A & 5'-CCCACGTTTATTTCACTAGATCC-3' \\
\hline ERG3-B & 5'-GGTTGCCTTGACCAACCC-3' \\
\hline ERG3-C & $5^{\prime}$-GGGAATGGGCAATAGGGACAC-3' \\
\hline ERG3-D & 5'-GGTTGCCTTGACCAACCC-3' \\
\hline \multicolumn{2}{|l|}{ C. albicans gene disruption } \\
\hline CaERG3A-F & 5'-ATCTGATTATATATGGGCCCAAGTGTTTG-3' \\
\hline CaERG3B-R & 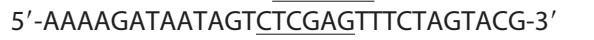 \\
\hline CaERG3C-F & 5'-ATAGCCGCGGTAACTCTTACAGAAGACC-3' \\
\hline CaERG3D-R & 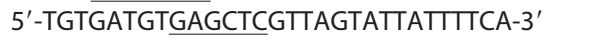 \\
\hline
\end{tabular}

aUnderlined sequences indicate introduced restriction sites. 
acetate, and $8 \mathrm{ml}$ of deionized water, and then reincubated at $30^{\circ} \mathrm{C}$ for $1 \mathrm{~h}$. Freshly prepared $1 \mathrm{M}$ dithiothreitol was added to the cell suspension, and the cells were incubated for an additional $30 \mathrm{~min}$. The cells were then washed twice with ice-cold water and then once with ice-cold $1 \mathrm{mM}$ sorbitol. Finally, the cells were resuspended in $100 \mu \mathrm{l}$ of fresh ice-cold $1 \mathrm{mM}$ sorbitol. The gel-purified Apal-Sacl fragment from the appropriate plasmid was mixed with $40 \mu \mathrm{l}$ of competent cells, and the mixture was transferred into a chilled 2-mm electroporation cuvette. The reaction was carried out at $1.5 \mathrm{kV}$, using a CelljecT Pro electroporator (Thermo). Immediately thereafter, $1 \mathrm{ml}$ of YPD containing $1 \mathrm{M}$ sorbitol was added and the mixture was transferred to a $1.5-\mathrm{ml}$ centrifuge tube. Cells were allowed to recover at $30^{\circ} \mathrm{C}$ for $6 \mathrm{~h}$. Finally, $100 \mu \mathrm{l}$ was removed and plated to YPD agar plates containing $200 \mu \mathrm{g} / \mathrm{ml}$ nourseothricin and $1 \mathrm{M}$ sorbitol. Transformants were selected after at least $48 \mathrm{~h}$ of growth at $30^{\circ} \mathrm{C}$.

RNA isolation and sequencing. RNA was isolated using the hot phenol method described previously (34). RNA concentrations were determined using a NanoDrop spectrophotometer (NanoDrop Products), and RNA integrity was verified using a Bioanalyzer 2100 instrument (Agilent Technologies). Barcoded libraries were prepared using a Lexogen mRNA Sense kit for lon Torrent according to the manufacturer's standard protocol. Libraries were sequenced on the lon Torrent Proton sequencer. Individual sample fragments were concatenated to form the fastq file for the whole sample. The files were then run through FastQC software to check the data quality. Any reads with a Phred score of $<20$ were trimmed. Reads were then aligned to the C. parapsilosis CDC317 reference transcriptome using the RNA-Star long method. After alignment, transcriptome alignment counts were gathered. The read counts for each sample were normalized using the transcripts per kilobase million (TPM) method.

Southern hybridization. Genomic DNA (gDNA) for use with Southern blotting was prepared as described previously (35), digested with appropriate restriction endonucleases, separated on a $1 \%$ agarose gel, stained with ethidium bromide, and transferred by vacuum blotting to a nylon membrane. After UV cross-linking, gDNA was detected using an Amersham ECL Direct nucleic acid labeling and detection system according to the manufacturer's instructions.

Whole-genome sequencing. Genomic DNA for library preparation was isolated using a Qiagen Genomic-tip 100/G kit (Qiagen) following the manufacturer's instructions for genomic DNA preparation. Paired-end DNA sequence libraries for isolate 1 and for isolate 2 were prepared and sequenced using the Ion Proton Torrent sequencer, and the sequences were aligned to the $C$. parapsilosis CDC317 reference genome sequence. The GATK Best Practices work flow was used, incorporating the following tools: bwa, v0.5.9 (aln and sample); Picard tools (http://picard.sourceforge.net), v1.107 (SortSam and MarkDuplicates); samtools, v0.1.19; and GATK, v2.8. (RealignerTargetCreateor, IndelRealigner, BaseRecalibrator, PrintReads, ReduceReads, HaplotypeCaller). A population variant file for C. parapsilosis was downloaded from the Broad Institute website (https://www.broadinstitute.org/fungal-genome-initiative) and used as the - knownSites file in the GATK BaseRecalibrator step. The snpEff, v3.5, genetic variant annotation tool was used to annotate SNP and indel variants using the c_parapsilosis_CDC317 snpEff database. The GATK CombineVariants and SelectVariants tools were used to select SNPs found in isolate 2 but not in isolate 1 and SNPs that were heterozygous in isolate 1 and homozygous in isolate 2 . The JBrowse Genome Browser, v1.11.6, was used to visualize sequence alignments at genomic positions in order to validate variant calls (36). Following installation of the C. parapsilosis CDC317 reference genome using the current chromosomal features file available at the Candida Genome Database (www .candidagenome.org), the whole-genome sequencing (WGS) data for the parental isolate 1 were uploaded and aligned to the reference chromosome map using Ymap (lovelace.cs.umn.edu/Ymap). WGS data for isolate 2 were then installed utilizing isolate 1 as the parental strain to allow loss-ofheterozygosity ( $\mathrm{LOH}$ ) analysis (16).

Sequence analysis of ERG3. The coding sequence of ERG3 in C. parapsilosis was amplified by PCR from C. parapsilosis genomic DNA using the primers listed in Table 5, cloned into pCR-BLUNTII-TOPO using a Zero Blunt TOPO PCR cloning kit, and transferred into Escherichia coli TOP10 cells with selection on LB agar plates containing $50 \mu \mathrm{g} / \mathrm{ml}$ kanamycin. Plasmid DNA was purified (QIAquick PCR purification kit; Qiagen) and sequenced on an $\mathrm{ABI}$ model 3130XL genetic analyzer using sequencing primers, resulting in a full-length sequence from both strands of ERG3. The sequencing was performed using a total of six sets of clones derived from three independent PCRs.

Sterol analysis. Nonsaponifiable lipids were extracted using alcoholic $\mathrm{KOH}$. Samples were dried in a vacuum centrifuge (Heto) and were derivatized by adding $100 \mu \mathrm{l} 90 \% \mathrm{~N}, \mathrm{O}$-bis(trimethylsilyl)trifluoroacetamide-10\% tetramethylsilane (TMS) (Sigma) and $200 \mu$ l anhydrous pyridine (Sigma) and heating for $2 \mathrm{~h}$ at $80^{\circ} \mathrm{C}$. TMS-derivatized sterols were analyzed and identified using GC-MS (with a Thermo 1300 GC coupled to a Thermo ISQ mass spectrometer; Thermo Scientific) with reference to the retention times and fragmentation spectra for known standards. GC-MS data files were analyzed using Xcalibur software (Thermo Scientific) to determine the sterol profiles for all isolates and for integrated peak areas (21).

Accession number(s). The RNA sequencing data have been deposited in the Gene Expression Omnibus repository under accession number GSE98986. The NCBI BioProject accession number for the WGS data is PRJNA361149. The coding sequence of ERG3 in isolate 2 described in this study has been deposited in GenBank under accession number KT277771.

\section{SUPPLEMENTAL MATERIAL}

Supplemental material for this article may be found at https://doi.org/10.1128/AAC .00651-17.

SUPPLEMENTAL FILE 1, XLSX file, $0.1 \mathrm{MB}$. 
SUPPLEMENTAL FILE 2, XLSX file, $0.1 \mathrm{MB}$.
SUPPLEMENTAL FILE 3, XLSX file, $0.1 \mathrm{MB}$.
SUPPLEMENTAL FILE 4, XLSX file, $0.1 \mathrm{MB}$.
SUPPLEMENTAL FILE 5, PDF file, $4.0 \mathrm{MB}$.

ACKNOWLEDGMENTS

We thank Elizabeth L. Berkow for her contributions to the construction of the $C$. parapsilosis ERG3 mutants used in this study, Joachim Morschhäuser for his invaluable expertise and for generously providing the pSFS2 plasmid used in the gene disruption and complementation experiments, and Shirlean Goodwin for her assistance with the improvement of genomic DNA isolation and sequencing methods.

The research contained in this report was supported by National Institutes of Health (NIH) grant R01 AI058145 (to P.D.R.). Partial support for DNA sequencing and analysis was provided by the Memphis Research Consortium (to T.R.S.).

\section{REFERENCES}

1. Pfaller MA, Andes DR, Diekema DJ, Horn DL, Reboli AC, Rotstein C, Franks B, Azie NE. 2014. Epidemiology and outcomes of invasive candidiasis due to non-albicans species of Candida in 2,496 patients: data from the Prospective Antifungal Therapy (PATH) registry 2004-2008. PLoS One 9:e101510. https://doi.org/10.1371/journal.pone.0101510.

2. Lockhart SR, lqbal N, Cleveland AA, Farley MM, Harrison LH, Bolden CB, Baughman W, Stein B, Hollick R, Park BJ, Chiller T. 2012. Species identification and antifungal susceptibility testing of Candida bloodstream isolates from population-based surveillance studies in two U.S. cities from 2008 to 2011. J Clin Microbiol 50:3435-3442. https://doi.org/10 .1128/JCM.01283-12.

3. Cleveland AA, Farley MM, Harrison LH, Stein B, Hollick R, Lockhart SR, Magill SS, Derado G, Park BJ, Chiller TM. 2012. Changes in incidence and antifungal drug resistance in candidemia: results from population-based laboratory surveillance in Atlanta and Baltimore, 2008-2011. Clin Infect Dis 55:1352-1361. https://doi.org/10.1093/cid/cis697.

4. Trofa D, Gacser A, Nosanchuk JD. 2008. Candida parapsilosis, an emerging fungal pathogen. Clin Microbiol Rev 21:606-625. https://doi.org/10 .1128/CMR.00013-08.

5. Garcia-Effron G, Katiyar SK, Park S, Edlind TD, Perlin DS. 2008. A naturally occurring proline-to-alanine amino acid change in Fks1p in Candida parapsilosis, Candida orthopsilosis, and Candida metapsilosis accounts for reduced echinocandin susceptibility. Antimicrob Agents Chemother 52:2305-2312. https://doi.org/10.1128/AAC.00262-08.

6. Kabbara N, Lacroix C, Peffault de Latour R, Socie G, Ghannoum M, Ribaud P. 2008. Breakthrough C. parapsilosis and C. guilliermondii blood stream infections in allogeneic hematopoietic stem cell transplant recipients receiving long-term caspofungin therapy. Haematologica 93:639-640. https://doi.org/10.3324/haematol.11149.

7. Pfeiffer CD, Garcia-Effron G, Zaas AK, Perfect JR, Perlin DS, Alexander BD. 2010. Breakthrough invasive candidiasis in patients on micafungin. J Clin Microbiol 48:2373-2380. https://doi.org/10.1128/JCM.02390-09.

8. Pfaller MA, Rhomberg PR, Messer SA, Jones RN, Castanheira M. 2015. Isavuconazole, micafungin, and 8 comparator antifungal agents' susceptibility profiles for common and uncommon opportunistic fungi collected in 2013: temporal analysis of antifungal drug resistance using CLSI species-specific clinical breakpoints and proposed epidemiological cutoff values. Diagn Microbiol Infect Dis 82:303-313. https://doi.org/10 .1016/j.diagmicrobio.2015.04.008.

9. Silva AP, Miranda IM, Guida A, Synnott J, Rocha R, Silva R, Amorim A, Pina-Vaz C, Butler G, Rodrigues AG. 2011. Transcriptional profiling of azole-resistant Candida parapsilosis strains. Antimicrob Agents Chemother 55:3546-3556. https://doi.org/10.1128/AAC.01127-10.

10. Grossman NT, Pham CD, Cleveland AA, Lockhart SR. 2015. Molecular mechanisms of fluconazole resistance in Candida parapsilosis isolates from a U.S. surveillance system. Antimicrob Agents Chemother 59: 1030-1037. https://doi.org/10.1128/AAC.04613-14.

11. Branco J, Silva AP, Silva RM, Silva-Dias A, Pina-Vaz C, Butler G, Rodrigues AG, Miranda IM. 2015. Fluconazole and voriconazole resistance in Candida parapsilosis is conferred by gain-of-function mutations in MRR1 transcription factor gene. Antimicrob Agents Chemother 59:6629-6633. https://doi.org/10.1128/AAC.00842-15.
12. Berkow EL, Manigaba K, Parker JE, Barker KS, Kelly SL, Rogers PD. 2015. Multidrug transporters and alterations in sterol biosynthesis contribute to azole antifungal resistance in Candida parapsilosis. Antimicrob Agents Chemother 59:5942-5950. https://doi.org/10.1128/AAC.01358-15.

13. Moudgal V, Little T, Boikov D, Vazquez JA. 2005. Multiechinocandin- and multiazole-resistant Candida parapsilosis isolates serially obtained during therapy for prosthetic valve endocarditis. Antimicrob Agents Chemother 49:767-769. https://doi.org/10.1128/AAC.49.2.767-769.2005.

14. National Committee for Clinical Laboratory Standards. 2002. Reference method for broth dilution antifungal susceptibility testing of yeasts; approved standard. CLSI document M27-A2. National Committee for Clinical Laboratory Standards, Wayne, PA.

15. Znaidi S, Weber S, Al-Abdin OZ, Bomme P, Saidane S, Drouin S, Lemieux S, De Deken X, Robert F, Raymond M. 2008. Genomewide location analysis of Candida albicans Upc2p, a regulator of sterol metabolism and azole drug resistance. Eukaryot Cell 7:836-847. https://doi.org/10.1128/ EC.00070-08.

16. Abbey DA, Funt J, Lurie-Weinberger MN, Thompson DA, Regev A, Myers $\mathrm{CL}$, Berman J. 2014. YMAP: a pipeline for visualization of copy number variation and loss of heterozygosity in eukaryotic pathogens. Genome Med 6:100. https://doi.org/10.1186/s13073-014-0100-8.

17. Prasad R, Rawal MK. 2014. Efflux pump proteins in antifungal resistance. Front Pharmacol 5:202. https://doi.org/10.3389/fphar.2014.00202.

18. Denning DW. 2003. Echinocandin antifungal drugs. Lancet 362:1142-1151. https://doi.org/10.1016/S0140-6736(03)14472-8.

19. Park S, Kelly R, Kahn JN, Robles J, Hsu MJ, Register E, Li W, Vyas V, Fan H, Abruzzo G, Flattery A, Gill C, Chrebet G, Parent SA, Kurtz M, Teppler H, Douglas CM, Perlin DS. 2005. Specific substitutions in the echinocandin target Fks1p account for reduced susceptibility of rare laboratory and clinical Candida sp. isolates. Antimicrob Agents Chemother 49: 3264-3273. https://doi.org/10.1128/AAC.49.8.3264-3273.2005.

20. Cowen LE, Steinbach WJ. 2008. Stress, drugs, and evolution: the role of cellular signaling in fungal drug resistance. Eukaryot Cell 7:747-764. https://doi.org/10.1128/EC.00041-08.

21. Kelly SL, Lamb DC, Corran AJ, Baldwin BC, Kelly DE. 1995. Mode of action and resistance to azole antifungals associated with the formation of 14 alpha-methylergosta-8,24(28)-dien-3 beta,6 alpha-diol. Biochem Biophys Res Commun 207:910-915. https://doi.org/10.1006/bbrc.1995.1272.

22. Sanglard D, Coste A, Ferrari S. 2009. Antifungal drug resistance mechanisms in fungal pathogens from the perspective of transcriptional gene regulation. FEMS Yeast Res 9:1029-1050. https://doi.org/10.1111/j.1567 -1364.2009.00578.x.

23. Martel CM, Parker JE, Bader O, Weig M, Gross U, Warrilow AG, Rolley N, Kelly DE, Kelly SL. 2010. Identification and characterization of four azole-resistant erg3 mutants of Candida albicans. Antimicrob Agents Chemother 54:4527-4533. https://doi.org/10.1128/AAC.00348-10.

24. Vale-Silva LA, Coste AT, Ischer F, Parker JE, Kelly SL, Pinto E, Sanglard D. 2012. Azole resistance by loss of function of the sterol delta(5),(6)desaturase gene (ERG3) in Candida albicans does not necessarily decrease virulence. Antimicrob Agents Chemother 56:1960-1968. https:// doi.org/10.1128/AAC.05720-11.

25. Hull CM, Bader O, Parker JE, Weig M, Gross U, Warrilow AG, Kelly DE, 
Kelly SL. 2012. Two clinical isolates of Candida glabrata exhibiting reduced sensitivity to amphotericin B both harbor mutations in ERG2. Antimicrob Agents Chemother 56:6417-6421. https://doi.org/10 .1128/AAC.01145-12.

26. Young LY, Hull CM, Heitman J. 2003. Disruption of ergosterol biosynthesis confers resistance to amphotericin B in Candida lusitaniae. Antimicrob Agents Chemother 47:2717-2724. https://doi.org/10.1128/AAC 47.9.2717-2724.2003.

27. Jensen RH, Astvad KM, Silva LV, Sanglard D, Jorgensen R, Nielsen KF, Mathiasen EG, Doroudian G, Perlin DS, Arendrup MC. 2015. Stepwise emergence of azole, echinocandin and amphotericin $B$ multidrug resistance in vivo in Candida albicans orchestrated by multiple genetic alterations. J Antimicrob Chemother 70:2551-2555. https://doi.org/10 $.1093 / \mathrm{jac} / \mathrm{dkv} 140$.

28. Kelly SL, Lamb DC, Kelly DE, Manning NJ, Loeffler J, Hebart H, Schumacher U, Einsele H. 1997. Resistance to fluconazole and cross-resistance to amphotericin B in Candida albicans from AIDS patients caused by defective sterol delta5,6-desaturation. FEBS Lett 400:80-82. https://doi .org/10.1016/S0014-5793(96)01360-9.

29. Chau AS, Gurnani M, Hawkinson R, Laverdiere M, Cacciapuoti A, McNicholas PM. 2005. Inactivation of sterol delta5,6-desaturase attenuates virulence in Candida albicans. Antimicrob Agents Chemother 49: 3646-3651. https://doi.org/10.1128/AAC.49.9.3646-3651.2005.

30. Miyazaki Y, Geber A, Miyazaki H, Falconer D, Parkinson T, Hitchcock $C$, Grimberg B, Nyswaner K, Bennett JE. 1999. Cloning, sequencing, expression and allelic sequence diversity of ERG3 (C-5 sterol desaturase gene) in Candida albicans. Gene 236:43-51. https://doi.org/10.1016/S0378-1119 (99)00263-2.

31. Branco J, Ola M, Silva RM, Fonseca E, Gomes NC, Martins-Cruz C, Silva AP, Silva-Dias A, Pina-Vaz C, Erraught C, Brennan L, Rodrigues AG, Butler G, Miranda IM. 11 February 2017. Impact of ERG3 mutations and expression of ergosterol genes controlled by UPC2 and NDT80 in Candida parapsilosis azole resistance. Clin Microbiol Infect. https://doi.org/10.1016/j .cmi.2017.02.002.

32. Reuss O, Vik A, Kolter R, Morschhauser J. 2004. The SAT1 flipper, an optimized tool for gene disruption in Candida albicans. Gene 341: 119-127. https://doi.org/10.1016/j.gene.2004.06.021.

33. Clinical and Laboratory Standards Institute. 2008. Reference method for broth microdilution antifungal susceptibility testing of yeasts; approved standard. CLSI document M27-A3, 3rd ed, vol 28. Clinical and Laboratory Standards Institute, Wayne, PA.

34. Schmitt ME, Brown TA, Trumpower BL. 1990. A rapid and simple method for preparation of RNA from Saccharomyces cerevisiae. Nucleic Acids Res 18:3091-3092. https://doi.org/10.1093/nar/18.10.3091.

35. Amberg DC, Burke DJ, Strathern JN. 2006. Isolation of yeast genomic DNA for Southern blot analysis. CSH Protoc https://doi.org/10.1101/pdb .prot4149.

36. Skinner ME, Uzilov AV, Stein LD, Mungall CJ, Holmes IH. 2009. JBrowse: a next-generation genome browser. Genome Res 19:1630-1638. https:// doi.org/10.1101/gr.094607.109. 